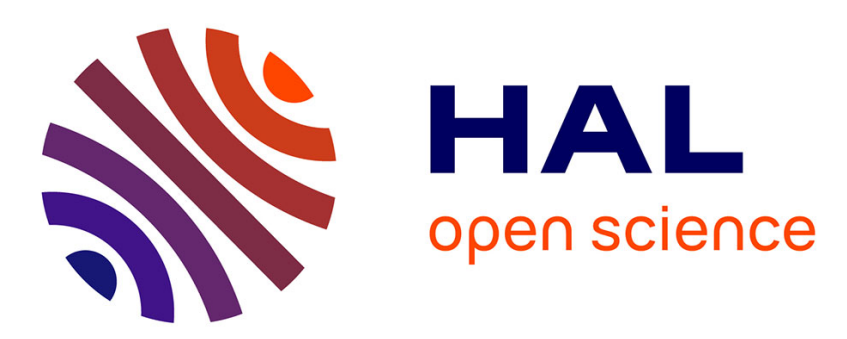

\title{
A heuristic for fast convergence in interference-free channel assignment using D1EC coloring
}

Fabio Campoccia, Vincenzo Mancuso

\section{To cite this version:}

Fabio Campoccia, Vincenzo Mancuso. A heuristic for fast convergence in interference-free channel assignment using D1EC coloring. ISCIS 2010, Sep 2010, LONDON, United Kingdom. inria-00506803

\section{HAL Id: inria-00506803 \\ https://hal.inria.fr/inria-00506803}

Submitted on 29 Jul 2010

HAL is a multi-disciplinary open access archive for the deposit and dissemination of scientific research documents, whether they are published or not. The documents may come from teaching and research institutions in France or abroad, or from public or private research centers.
L'archive ouverte pluridisciplinaire HAL, est destinée au dépôt et à la diffusion de documents scientifiques de niveau recherche, publiés ou non, émanant des établissements d'enseignement et de recherche français ou étrangers, des laboratoires publics ou privés. 


\title{
A heuristic for fast convergence in interference-free channel assignment using D1EC coloring
}

\author{
Fabio Campoccia ${ }^{1}$, Vincenzo Mancuso ${ }^{2}$ \\ ${ }^{1}$ Università degli studi di Palermo, Italy, ${ }^{2}$ INRIA Sophia Antipolis, France
}

\begin{abstract}
This work proposes an efficient method for solving the Distance-1 Edge Coloring problem (D1EC) for the assignment of orthogonal channels in wireless networks with changing topology. The coloring algorithm is performed by means of the simulated annealing method, a generalization of Monte Carlo methods for solving combinatorial problems. We show that the simulated annealing-based coloring converges fast to a suboptimal coloring scheme. Furthermore, a stateful implementation of the D1EC scheme is proposed, in which network coloring is executed upon topology changes. The stateful D1EC reduces the algorithm's convergence time by one order of magnitude in comparison to stateless algorithms.
\end{abstract}

Keywords: Channel assignment, Edge coloring, Simulated annealing.

\section{Introduction}

Tremendous growth of 802.11 wireless networks in the last years allows adopting protocols that optimize the usage of the narrow available radio spectrum. In particular, the main problem that degrades network performance is the interference between concurrent transmissions, i.e., packet collisions. To avoid collisions, it is necessary to coordinate the transmitters within the network to access the wireless media through a multiple access scheme based on time, code or frequency. The availability of orthogonal channels (e.g., in CDMA or OFDMA systems) allows to assign dedicated channels to node pairs that would otherwise interfere with each other, so that multiple simultaneous transmissions can successfully occur. However, the number of available orthogonal channels is limited. Thereby, it is not always possible to assign a different channel to each different node pair.

Channel assignment can be seen as a graph coloring problem [1], by using, e.g., the "distance-1 edge coloring" (D1EC) described in [2]. We propose a heuristic for D1EC which uses a centralized algorithm and exploits a global knowledge of the network topology and the node activity. Our channel assignment algorithm uses a combinatorial method called simulated annealing [3]. In particular, we propose a stateful approach to further improve the coloring algorithm performance by running the coloring scheme from an initial channel assignment whose cost is near to the minimum. We developed a Java simulator to validate our proposal.

\subsection{Definitions}

Here we recall the basic definitions about graph coloring and simulation annealing.

Graph coloring. It consists in assigning a color to each edge of a graph, so that no two adjacent edges share the same color. A k-coloring is an assignment of edges to $k$ colors. We say that a graph $G$ is $k$-colorable if there exists a k-coloring for $G$. Given a $\mathrm{k}$-colorable graph $G$, finding a k-coloring is solvable in polynomial time for $k=2$, but 
NP-hard for $k \geq 3$ [4]. Since graph coloring is NP-complete, the channel assignment problem is also NP-complete, and therefore an optimal assignment cannot be found in polynomial time. Several approaches have been developed to define a channel assignment problem as a graph coloring problem, e.g., in cellular radio networks [56], and in IEEE 802.11-based mesh networks [7-8].

Link distance. Given a graph $G$, and two nodes $u$ and $v$ contained in $G$, a link $l_{u v}$ is defined as the edge contained in $G$ that connects node $u$ to node $v$. Let the distance $d\left(u_{1}, u_{2}\right)$ between two nodes $u_{1}$ and $u_{2}$ in a graph $G$ be the minimum number of hops in $G$ from $u_{1}$ to $u_{2}$. Then the distance between two links $l_{u 1 u 2}$ and $l_{v 1 v 2}$ is the minimum of distances between one node in $\left\{u_{1}, u_{2}\right\}$ and one node in $\left\{v_{1}, v_{2}\right\}$.

Hence, zero-distance between two links means that the two links share a common node; distance one means that at least one edge connects one node of the first link to one node in the second link, hence simultaneous transmissions interfere. Distances greater than one are achieved if no edges connect a node in the first link to a node in the second link. So, channels can be reused over links whose distance is two or more.

D1EC channel assignment. Given a graph $G$ and a sub-graph $A \subseteq G$, "the Distance1 Edge Coloring (D1EC) problem seeks a mapping of colors to links in $A$ such that any two links in $A$ that are at distance one with respect to $G$ are assigned different colors" [2]. The authors of [2] do not discuss the time needed for the coloring to be computed and show the network throughput for a colored static topology, i.e., a static channel assignment is calculated before starting the experiments.

Simulated annealing. It was originally proposed by Kirkpatrick et al. [3] and Cerny [9]: a system has a set $S$ of possible conformations $s$, each having a cost $K(s)$ and a set of neighbors $N(s)$ representing the possible state transitions from $s$. A transition from $s$ to $s^{\prime} \in N(s)$ occurs according to the Metropolis criterion [10], i.e.: if the cost difference $\Delta K=K\left(s^{\prime}\right)-K(s)$ is negative then the $s^{\prime}$ becomes the new conformation; else, a random number $R$ between 0 and 1 is generated according to a uniform distribution, and the resulting conformation is accepted if $e^{-\Delta K / C}>R$. The parameter $C$ is a constant called control parameter. It regulates the percentage of new configurations that are accepted in case $\Delta K$ is positive. A cooling process, in simulated annealing applications, is emulated by decreasing $C$ progressively from $C_{0}$ to $C_{f}$ through a function $f(C)=u C$. Note that $C_{0}$ is critical in order to engage the simulating annealing process, while $C_{f}$ allows to define a final condition for the algorithm.

\section{Dynamic channel assignment through simulated annealing}

An active network topology is a subnet that only contains nodes that are sending and receiving packets, and edges that connect these nodes. If the active network changes, also the interference map changes. Furthermore, static coloring of the entire network results in using an unnecessary high number of colors to assign channels to edges that will not really contend most of the time. In practice, with a dynamic coloring scheme, channel assignment could be performed for the sub-graph comprising the active nodes only, and online, e.g., when the set of active nodes changes or on a fixed schedule. To speed-up the online coloring update procedure, we further exploit the fact that, upon topology changes, the current coloring is likely to be close to the optimal coloring for the new active topology. Hence we run D1EC via simulated annealing as soon as the traffic matrix changes. The contention degree, i.e., the number of interfering links, 
represents the cost in the Metropolis criterion. We developed a Java simulator to evaluate the coloring scheme, as well as to design and test the simulated annealing parameters. The details about the simulator and the Java code are omitted due to space constraints. Our simulations show that applying D1EC to the set of active nodes is efficient, and initializing D1EC with the colors in use in the previous subinterval converges faster than starting from a non-colored graph (stateful initial condition).

\subsection{Design of a D1EC-suitable simulated annealing}

Different perturbation methods are used for promoting state transitions in simulated annealing and obtain a fast convergence to a coloring conformation which is close to the optimal one. Since the algorithm tuning depends on the network topology, we considered different topologies for which we found similar results. Hence we only show results for the network of Fig. 1, which is an extraction from the Chaska's 802.11 network, and contains 45 vertex and 75 edges. As baseline, we considered an algorithm that does not use the Metropolis criterion, and hence accept state transitions only if $\Delta K>0$. We call this algorithm

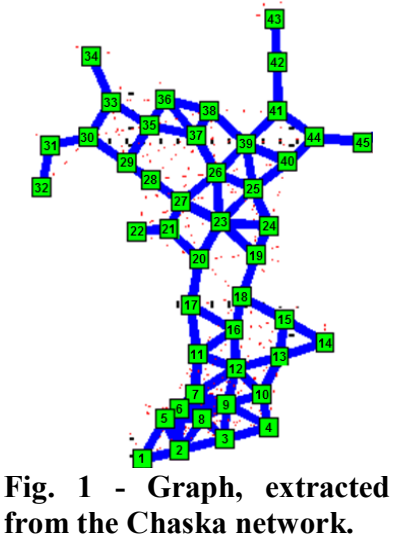
NOT-SA, and we compared its performance to the one of a legacy perturbation method (SA) in which we $(i)$ select all edge pairs that have the same color and whose distance is one, ( $i$ i) assign a random color to those edge, and (iii) use the Metropolis criterion. Following [3] we found that the optimal values for $C_{0}$ is 4 , and $u=0.95 . C_{f}$ was set such that SA effectively converges. Results-not shown here due to lack of space-show that SA outperforms NON-SA. In particular, SA reaches a significant cost reduction within little iteration, while NON-SA converges more slowly.

\section{Evaluation}

All tests reported here were repeated 10 times, and results are shown in terms of average statistics. We first consider a stateless version of our algorithm, and then we show that the stateful algorithm is ten times faster than the stateless one. In order to show the advantage of using the stateful initial condition, we simulate D1EC with simulation annealing in a network in which one node pair is activated after the other, following a random order. When a node pair is activated, the shortest path between the two nodes is computed, and all nodes and links on the path are added to the active network topology. This way, we simulate the impact of multihop wireless traffic.

In Fig. 2, we report the performance of the D1EC when all computations are initialized with a monochromatic assignment, and 3 to 19 colors are available for D1EC. In the figure, the cost decreases dramatically with respect to the initial cost, and fifty iterations are enough to reduce the cost to less than $10 \%$ of the initial cost when using at least seven colors. With three colors, the network under test cannot be colored with zero cost. Anyway, also in that case, the minimum possible cost is approached in as few as fifty iterations. Further iterations do not really reduce the cost remarkably. The convergence time is shown in Fig. 3, which reports the number of iterations required to converge after a node pair is added to the active topology: the $\mathrm{x}$ - 


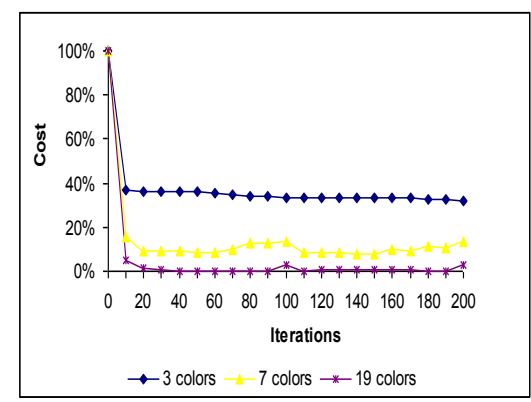

Fig. 2 - Cost convergence (all nodes).

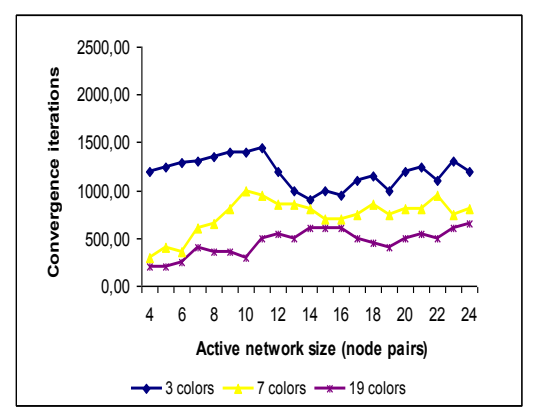

Fig. 3 - Convergence time (stateless).

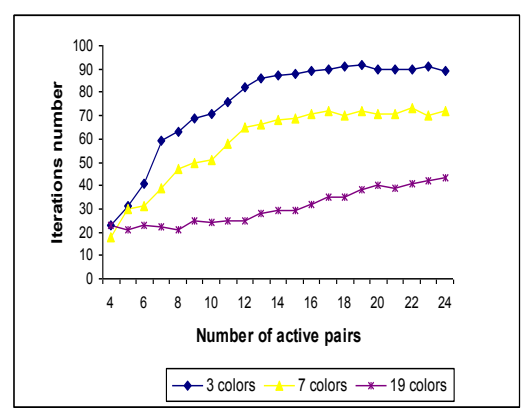

Fig. 4 - Convergence time (stateful).

axis represents the number of node pairs already in the network when the topology changes, and the y-axis shows the iterations to convergence after the topology change. A few hundreds of iterations are needed in order to converge at each step. In the results of Fig. 4, D1EC uses the last active coloring scheme as initial coloring for the updated network graph, and newly added edges are initialized with a same random color. Noticeably, whatever the number of available colors, the stateful algorithm converges in few tens of iterations, ten times faster than the stateless algorithm.

\section{Conclusions}

We proposed a simulated annealing-based dynamic channel assignment algorithm, and demonstrated that it can quickly compute a good suboptimal coloring. In particular, we introduced a state in the algorithm, which makes the coloring ten times faster by using an initial channel assignment whose cost is near to the minimum.

\section{References}

1. W. K. Hale, "Frequency assignment: Theory and applications." In Proc. IEEE, vol. 68, pp. 1497-1514, Dec. 1980.

2. E. Aryafar, O. Gurewitz, E.W. Knightly, "Distance-1 Constrained Channel Assignment in Single Radio Wireless Mesh Networks." In Proc. of IEEE INFOCOM 2008.

3. S. Kirkpatrick, C.D. Gelatt Jr., M.P. Vecchi, "Optimization by simulated annealing," Science, vol. 220, no. 4598, pp. 671-680, May 13, 1983.

4. Chun-Chen Hsu, Pangfeng Liu, Da-wei Wang, Jan-Jan Wu, "Generalized Edge Coloring for Channel Assignment in Wireless Networks.” In Proc. of ICPP 2006, pp. 82 - 92.

5. R. Battiti, A. Bertossi, D. Cavallaio, "A Randomized Saturation Degree Heuristic for Channel Assignment in Cellular Radio Networks." IEEE Trans. on Vehicular Technology, Vol. 50, issue 2, pp. 364-374, 2001.

6. M.I. Islam, A.B.M. Siddique Hossain, "Channel Allocation of Mobile Cellular Network Based on Graph Theory.” In Proc. of IEEE TENCON 2004, 21-24 November 2004.

7. J. Riihijarvi, M. Petrova, P. Mahonen, "Frequency Allocation for WLANs Using Graph Colouring Techniques." In Proc. of the WONS 2005, pp. 216-222, January 19-21, 2005

8. C.L. Barrett, V.S.A. Kumar, M.V. Marathe, S. Thite, G. Istrate, "Strong Edge Coloring for Channel Assignment in Wireless Radio Networks.” In Proc. of PERCOMW 2006, p. 106.

9. V. Cerny, "Thermodynamical approach to the travelling salesman problem: an efficient simulation algorithm.” Journal on Opt. Theory Appl., vol. 45, pp. 41-51, 1985.

10. N. Metropolis, A. Rosenbluth, M. Rosenbluth, A. Teller, E. Teller, "Equation of state calculations by fast computing machines.” Jour. of Ch. Phys., vol. 21, pp. 1087-1092, 1953. 